\title{
Numerical Simulation of EIT-Based Slow Light in the Doppler-Broadened Atomic Media of the Rubidium D2 Line
}

\author{
Yi Chen ${ }^{1}$, Xiao Gang Wei ${ }^{2}$ and Byoung Seung Ham ${ }^{3}$ \\ ${ }^{1}$ Institute of Atomic and Molecular Physics, Jilin University \\ ${ }^{2}$ College of Physics, Jilin University \\ ${ }^{3}$ School of Electrical Engineering, Inha University \\ 1,2China \\ ${ }^{3}$ South Korea
}

\section{Introduction}

Quantum coherence and interference effects (Scully \& Zubairy, 1997) in atomic systems have attracted great attention in the last two decades. With quantum coherence, the absorption and dispersion properties of an optical medium can be extremely modified, and can lead to many important effects such as coherent population trapping (CPT) (Arimondo \& Orriols, 1976; Alzetta et al., 1976; Gray et al., 1978), lasing without inversion (LWI) (Harris, 1989; Scully et al., 1989; Padmabandu et al., 1996), electromagnetically induced transparency (EIT) (Boller et al., 1991; Harris, 1997; Ham et al., 1997; Phillips et al., 2003; Fleischhauer et al., 2005; Marangos, 1998), high refractive index without absorption (Scully, 1991; Scully \& Zhu, 1992; Harris et al., 1990), giant Kerr effect (Schmidt \& Imamoglu, 1996), slow and fast light (Boyd \& Gauthier, 2002), light storage (Phillips et al., 2001), and other effects. In particular, EIT plays an important role in the quantum optics area.

EIT, named by Harris and his co-workers, has been extensively studied both experimentally and theoretically since it was proposed in 1990 (Harris et al., 1990). Harris et al. first experimentally demonstrated EIT in Sr atomic vapour in 1991 (Boller et al., 1991), providing the basis for further EIT works. Subsequently, M. Xiao and co-workers successfully observed the EIT effect in $\mathrm{Rb}$ vapor by using continuous wave (CW) diode lasers (Xiao et al., 1995; Li \& Xiao, 1995). This work simplified EIT research, and attracted related research. With the growth of EIT technique, the researchers also realized EIT in several solid state materials and semiconductors (Serapoglia et al., 2000; Zhao et al., 1997; Ham et al., 1997). These works provide a firm foundation for EIT-based applications.

One of EIT applications is slow light. Due to the steep dispersion property within the EIT transparency window, EIT can be used to control the group velocity of light. In the past decade, ultraslow group velocity based on EIT (Harris et al., 1992) has drawn much attention to quantum optical applications, such as quantum memories (Liu et al., 2001; Turukhin et al., 2002; Julsgaard et al., 2004), quantum entanglement generations (Lukin \& Hemmer, 2000; Petrosyan \& Kurizki, 2002; Paternostro et al., 2003), quantum routing (Ham, 2008), and quantum information processing (Nielsen \& Chuang, 2000). So far EIT-based slow light has 
been observed in many media. In 1995, S. E. Harris and co-workers observed group velocity as slow as c /165 in Pb vapour (Kasapi et al., 1995). In 1999, Hau et al. obtained the famous ultraslow group velocity $17 \mathrm{~m} / \mathrm{s}$ in Bose-Einstein condensate of $\mathrm{Na}$ (Hau et al., 1999). In the same year, Scully et al. reported the group velocity of $90 \mathrm{~m} / \mathrm{s}$ in hot rubidium gas $(360 \mathrm{~K})$ (Kash et al., 1999). In 2002, the light speed of $45 \mathrm{~m} / \mathrm{s}$ was demonstrated in an optically dense crystal of Pr doped $\mathrm{Y}_{2} \mathrm{SiO}_{5}$ by B. S. Ham et al. (Turukhin et al., 2002).

Based on deeply investigated EIT and slow light in simple three-level system, recently, researchers have turned their interests to multi-level system, which may render more interesting phenomena and closer to the realistic situations. In this chapter, we will study EIT and EIT-based slow light in a Doppler-broadened six-level atomic system of the rubidium D2 line. This research work may offer a clearer understanding of the slow light phenomenon in the complicated multi-level system, and also present a system whose hyperfine states are closely spaced within the Doppler broadening for potential applications of optical and quantum information processing, such as multichannel all-optical buffer memories and slow-light-based enhanced cross-phase modulation (Petrosyan \& Kurizki, 2002; Paternostro et al., 2003).

This chapter is organized as following: In section 2, we brief review EIT in a three-level system and discuss EIT in a Doppler-broadened multi-level atomic system of the rubidium D2 line. In section 3, based on the results we obtained in section 2, we study EIT-based slow light in the same atomic system. In section 4 , we introduce an N-type system, and numerically simulate slow light phenomenon in such kind of system. Finally, section 5 offers conclusions.

\section{EIT in the Doppler-broadened multi-level atomic system of ${ }^{87} \mathrm{Rb} \mathrm{D} 2$ line}

\subsection{Brief review of EIT in a three-level system}

EIT is one of the most important quantum coherent effects, and also serves as the foundation of this chapter. We will first review the optical properties of EIT in a three-level system. Fig. 1 shows the most famous three types of EIT scheme: lambda, ladder, and vee. Among these three EIT types, the lambda type is the best candidate to obtain EIT and EIT-related effects. For this reason, we will illustrate the EIT phenomenon by using the lambda configuration.

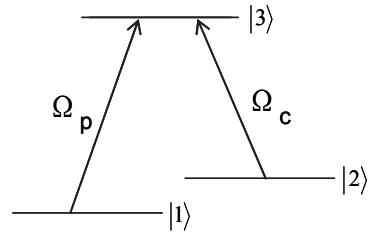

(a)

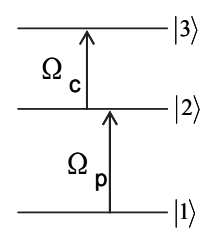

(b)

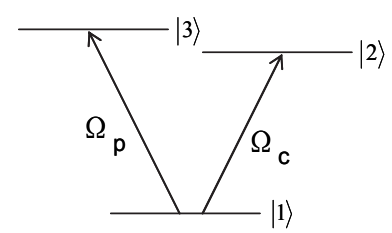

(c)

Fig. 1. Schematic of EIT in (a) lambda, (b) ladder, and (c) vee-type schemes.

In the absence of the coupling field $\Omega_{\mathrm{c}}$ absorption of the probe field is described by the blue curve in Fig. 2(a). When the probe frequency is resonant with the transition $|1\rangle-|3\rangle$, the probe field is strong absorbed by the medium. When we add a coupling field to the system, the strong absorbed peak of the probe disappears at the resonant frequency due to this coupling field (red curve in Fig. 2(a)). This means that the coupling field can modify the absorption property of the medium, and make the optically medium transparent. The transparent position depends on the detuning of the coupling field, and the transparent 
degree is determined by the Rabi frequency of the coupling field. The physics underlying the EIT can be clearly explained by the dressed state theory (Scully \& Zubairy, 1997): almost zero absorption at the resonant frequency is due to the destructive interference between two channels. Except for making the opaque media transparent, the steep dispersion characteristic at the resonant region (see red curve in Fig. 2(b)) is another important feature of EIT. This steep dispersion characteristic allows for control of the group velocity of the light, and opens up a series of promising applications.
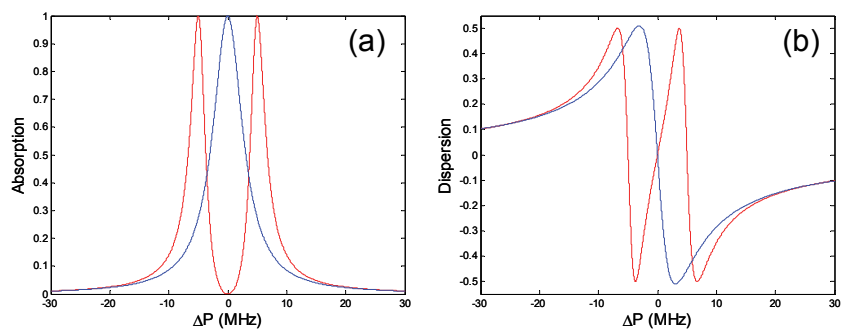

Fig. 2. (a) Absorption and (b) dispersion of probe field as a function of probe detuning for a three-level lambda system.

\subsection{EIT in the multi-level atomic system of ${ }^{87} \mathrm{Rb}$ D2 line}

Based on the theory of EIT in a three-level system, now we can study the EIT phenomenon in a multi-level atomic system.

\subsubsection{Model and theory}

An energy level diagram of the ${ }^{87} \mathrm{Rb} \mathrm{D} 2$ line is shown in Fig. 3. It shows a six-level atomic system, where $F=1(\mid 1>)$ and $F=2(\mid 2>)$ of $5 S_{1 / 2}$ form two ground levels and $F^{\prime}=0,1,2,3$ $(|3>| 4\rangle,,|5\rangle,|6\rangle)$ of $5 P_{3 / 2}$ form excited levels. The coupling field with frequency $\omega_{c}$ and amplitude $E_{\mathrm{c}}$ couples the levels $\mid 4>$ and $\mid 2>$, while the probe field with frequency $\omega_{p}$ and amplitude $E_{p}$ couples the levels $\mid 4>$ and $\mid 1>$. The frequency detuning of the coupling and probe is given by $\Delta_{c}=\omega_{c}-\omega_{42}$ and $\Delta_{p}=\omega_{p}-\omega_{41}$, respectively. Thus, a typical $\Lambda$-type EIT scheme can be satisfied.

In the framework of semiclassical theory, the Hamiltonian for this scheme is given by $H=H_{0}+H_{1}$, where $H_{0}$ and $H_{1}$ represent the unperturbed and interaction parts of the Hamiltonian, respectively. The interaction Hamiltonian $H_{1}$ can be written as:

$$
\begin{aligned}
H_{1}= & -\frac{\hbar}{2}\left(\Omega_{p 31} e^{-i \omega_{p} t}|3\rangle\left\langle 1\left|+\Omega_{p 41} e^{-i \omega_{p} t}\right| 4\right\rangle\left\langle 1\left|+\Omega_{p 51} e^{-i \omega_{p} t}\right| 5\right\rangle\langle 1|\right. \\
& \left.+\Omega_{c 42} e^{-i \omega_{c} t}|4\rangle\left\langle 2\left|+\Omega_{c 52} e^{-i \omega_{c} t}\right| 5\right\rangle\left\langle 2\left|+\Omega_{c 26} e^{-i \omega_{c} t}\right| 6\right\rangle\langle 2|+H . C .\right)
\end{aligned}
$$

where $\Omega_{p i 1}=\mu_{i 1} E_{p} / \hbar$ is the Rabi frequency of the probe field for the transition $\left.|\mathrm{i}>-| 1\right\rangle$ (i $=3,4,5)$, and $\Omega_{C j 2}=\mu_{j 2} E_{C} / \hbar$ is the Rabi frequency of the coupling field for the transition $|\mathrm{j}\rangle$ $-|2\rangle(j=4,5,6)$. For the ${ }^{87} \mathrm{Rb} \mathrm{D} 2$ line, the transitions $5 \mathrm{~S}_{1 / 2}, \mathrm{~F}=2 \rightarrow 5 \mathrm{P}_{3 / 2}, \mathrm{~F}^{\prime}=0$ and $5 \mathrm{~S}_{1 / 2}$, $\mathrm{F}=1 \rightarrow 5 \mathrm{P}_{3 / 2}, \mathrm{~F}^{\prime}=3$ are forbidden.

Under the rotating-wave approximation, the density matrix equation of motion for the interaction Hamiltonian is described by: 


$$
\dot{\rho}=-\frac{i}{\hbar}\left[H_{I}, \rho\right]-\frac{1}{2}\{\Gamma, \rho\} .
$$

The susceptibility $\chi\left(\Delta_{p}\right)=\chi^{\prime}+i \chi^{\prime \prime}$ can be obtained by solving this density matrix equation numerically under the steady state condition, where $\chi^{\prime}$ and $\chi^{\prime \prime}$ represent dispersion and absorption, respectively. Under the Doppler broadening which resulted from the random motions of atoms, the total susceptibility for all excited levels becomes:

$$
\chi_{\text {Dop }}\left(\Delta_{P}\right)=\int_{-\infty}^{\infty} \chi\left(\Delta_{P}, v\right) \frac{N}{v_{p} \sqrt{\pi}} e^{-v^{2} / v_{p}{ }^{2}} d v
$$

where $N$ is the atom density, $v_{p}=\sqrt{2 k T / m}=\sqrt{2 R T / M}$ is the most probable atom velocity, $\mathrm{k}$ is the Boltzmann constant, $\mathrm{R}$ is the gas constant, and $\mathrm{T}$ is the temperature of the atomic system. In Eq. (3), $\Delta_{P}$ and $\Delta_{C}$ are substituted by $\Delta_{P}-\omega_{41} v / c$ and $\Delta_{C}-\omega_{42} v / c$, respectively.

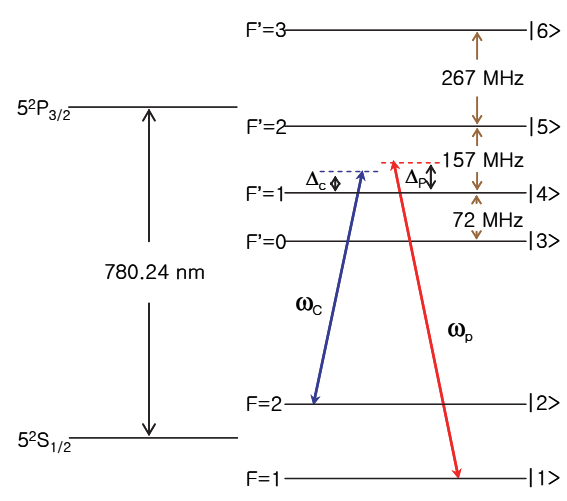

Fig. 3. Schematic diagram of a six-level ${ }^{87} \mathrm{Rb}$ (D2 line) atomic system for EIT.

\subsubsection{Numerical simulations and discussion}

We consider the following cases:

Case I: The coupling laser is resonant with the transition $5 \mathrm{~S}_{1 / 2}, \mathrm{~F}=2 \rightarrow 5 \mathrm{P}_{3 / 2}, \mathrm{~F}^{\prime}=1(|2>-| 4>)$;

Case II: The coupling laser is resonant with the center line between the level $5 \mathrm{P}_{3 / 2}, \mathrm{~F}^{\prime}=1$ and $5 \mathrm{P}_{3 / 2}, \mathrm{~F}^{\prime}=2$ from level $5 \mathrm{~S}_{1 / 2}, \mathrm{~F}=2$;

Case III: The coupling laser is resonant with the transition $5 S_{1 / 2}, F=2 \rightarrow 5 P_{3 / 2}, F^{\prime}=2(|2>-| 5>)$;

Case IV: The coupling laser is resonant with the center line between the level $5 \mathrm{P}_{3 / 2}, \mathrm{~F}^{\prime}=1$ and $5 \mathrm{P}_{3 / 2}, \mathrm{~F}^{\prime}=3$ from level $5 \mathrm{~S}_{1 / 2}, \mathrm{~F}=2$;

Case V: The coupling laser is resonant with the center line between the level $5 \mathrm{P}_{3 / 2}, \mathrm{~F}^{\prime}=2$ and $5 \mathrm{P}_{3 / 2}, \mathrm{~F}^{\prime}=3$ from level $5 \mathrm{~S}_{1 / 2}, \mathrm{~F}=2$;

Case VI: The coupling laser is resonant with the transition $5 S_{1 / 2}, F=2 \rightarrow 5 P_{3 / 2}, F^{\prime}=3(|2>-| 6>)$.

Based on the density matrix equations obtained in the previous subsection, we can numerically calculate the Doppler-broadened absorption (Fig. 4(a)) and dispersion (Fig. $4(b))$ of the probe for a particular transition with different Rabi frequencies of the coupling field, where the coupling (probe) is tuned to the transition $|4>-| 2\rangle(|4\rangle-|1\rangle)$ in case I. The parameters used in Fig. 4 are $\mathrm{T}=50^{\circ} \mathrm{C}, \Gamma_{21}=0.3 \mathrm{MHz}, \Gamma_{31}=6 \mathrm{MHz}, \Gamma_{41}=5 \mathrm{MHz}$, $\Gamma_{51}=3 \mathrm{MHz}, \Gamma_{42}=1 \mathrm{MHz}, \Gamma_{52}=3 \mathrm{MHz}, \Gamma_{62}=6 \mathrm{MHz}, \Omega_{C 42}=\sqrt{1 / 20} \Omega_{C}, \Omega_{C 52}=1 / 2 \Omega_{C}$, $\Omega_{C 62}=\sqrt{7 / 10} \Omega_{C}, \Delta_{34}=72 \mathrm{MHz}, \Delta_{45}=157 \mathrm{MHz}, \Delta_{56}=267 \mathrm{MHz}$, and $\Delta_{c}=0 \mathrm{MHz}$. 
Unlike the Doppler-free case in an ideal three-level system, where EIT line center locates at two-photon resonance frequency, EIT detuning exists in the multilevel system of Fig. 3, even with a small coupling Rabi frequency much less than the separation between the nearest neighboring state $|3\rangle$ (see the inset of Fig. 4(a)). When the Rabi frequency of the coupling increases, the EIT linewidth becomes wider. In particular, the EIT position is variable for different Rabi frequencies, whereas in a three-level system, it is not. As the Rabi frequency of the coupling field increases, the EIT position becomes more red-shifted, due to the extra interactions with the neighboring excited levels and the different dipole moment between different transitions.
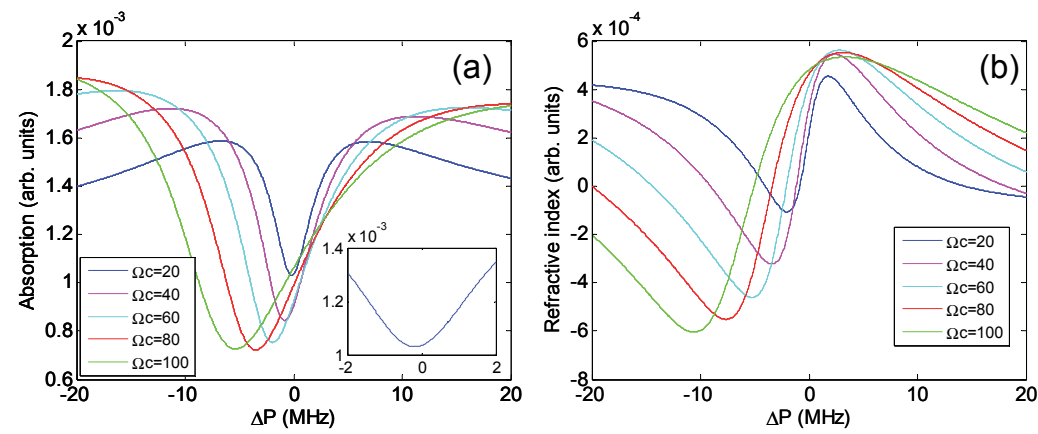

Fig. 4. The absorption (a) and dispersion spectra (b) for a six-level Doppler-broadened system (Case I).

In exploring this phenomenon further, we neglect the level $|6\rangle$ in the structure shown in Fig. 3 and assume that the transition $|3\rangle-|2\rangle$ is allowed for the coupling field. Furthermore, we assume the neighboring levels are symmetrically distributed $\left(\triangle_{34}=\triangle_{45}=72\right.$ $\mathrm{MHz}$ ). By setting the same decay rates and the same dipole moments for all transitions $\left(\Omega_{C 32}=\Omega_{C 42}=\Omega_{C 52}=40 \mathrm{MHz}\right)$, the system becomes symmetrical. There is no EIT detuning in this system, as shown in Fig. $5(\mathrm{a})$. In the $87 \mathrm{Rb} \mathrm{D} 2$ line, the level $5 \mathrm{P}_{3 / 2}, \mathrm{~F}^{\prime}=0$ is much nearer the level $5 \mathrm{P}_{3 / 2}, \mathrm{~F}^{\prime}=1$ than the level $5 \mathrm{P}_{3 / 2}, \mathrm{~F}^{\prime}=2\left(\triangle_{34}=72 \mathrm{MHz}, \triangle_{45}=157 \mathrm{MHz}\right)$. Under this condition, and keeping all the decay rates and dipole moments the same, we find that the EIT position becomes red shifted (Fig. 5(b)). However, if we assume unbalanced dipole moments $\left(\mu_{52}=2 \mu_{32}=2 \mu_{42}, \Omega_{C 32}=\Omega_{C 42}=\Omega_{C 52} / 2=40 \mathrm{MHz}\right)$ for the neighboring levels symmetrically distributed $\left(\Delta_{34}=\Delta_{45}=72 \mathrm{MHz}\right)$, we also find that the EIT position is red shifted as shown in Fig. 5(c). If we use another unbalanced dipole moments condition $\left(\mu_{32}=2 \mu_{52}=2 \mu_{42}\right)$, then the EIT position becomes blue shifted as shown in Fig. 5(d).

By using the parameters in the ${ }^{87} \mathrm{Rb}$ D2 line, for Cases I through VI (I: $\triangle_{c}=0 \mathrm{MHz}$; II: $\triangle_{c}=157 / 2 \mathrm{MHz} ;$ III: $\triangle_{c}=157 \mathrm{MHz}$ IV: $\triangle_{c}=157+267 / 2 \mathrm{MHz} ; \mathrm{V}: \triangle_{c}=(157+267) / 2 \mathrm{MHz}$; VI: $\left.\triangle_{c}=157+267 \mathrm{MHz}\right)$, we calculate the Doppler broadened absorption of the probe field as a function of one-photon detuning for corresponding $\Delta_{c}$. As shown in Fig. 6, EIT red detuning always occurs, because in the ${ }^{87 \mathrm{Rb}} \mathrm{D} 2$ line, the relative dipole matrix elements are $\sqrt{1 / 20}, 1 / 2, \sqrt{7 / 10}$ for the transitions $|2\rangle-|4\rangle,|2\rangle-|5\rangle$ and $|2\rangle-|6\rangle$, and the neighboring levels are unsymmetrically distributed $\left(\triangle_{34}=72 \mathrm{MHz}, \triangle_{45}=157 \mathrm{MHz}, \triangle_{56}=267\right.$ $\mathrm{MHz}$ ). In Fig. 6, the Rabi frequency of the coupling field is $\Omega_{C}=80 \mathrm{MHz}$, and other parameters are same as those in Fig. 4. 

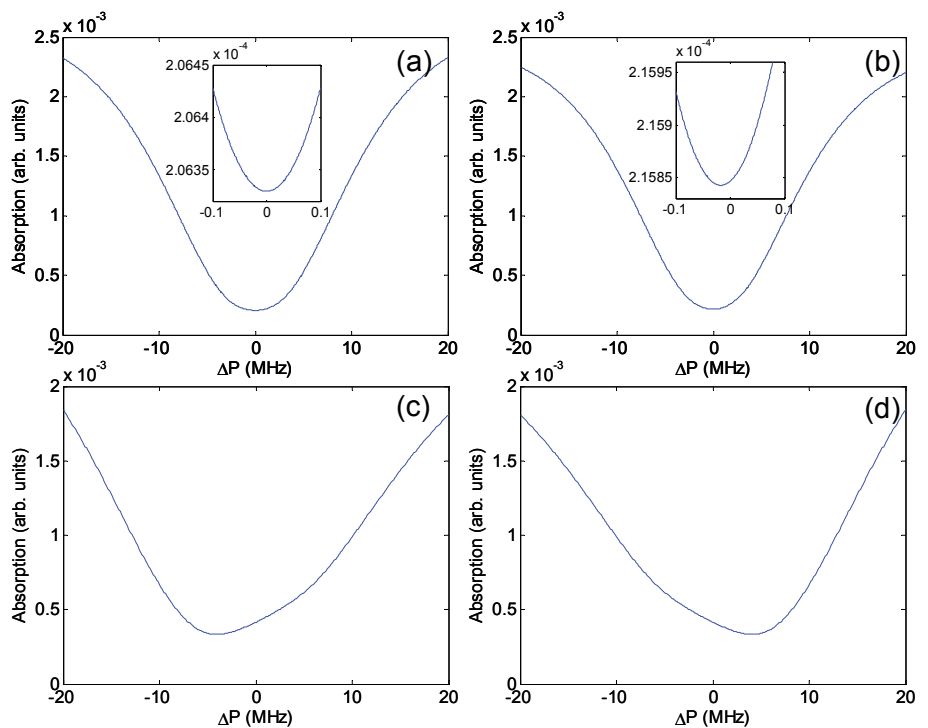

Fig. 5. The absorption spectra for a five-level Doppler-broadened system.

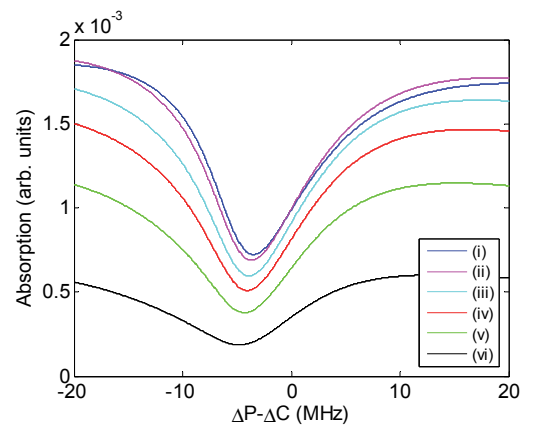

Fig. 6. The absorption spectra for a six-level Doppler-broadened system for Cases I $\sim$ VI.

\section{EIT-based slow light in the multi-level atomic system of ${ }^{87} \mathrm{Rb}$ D2 line}

Because of the steep dispersion spectrum directly resulting from the narrower EIT window according to the Kramers Kronig relation, the group velocity of the probe pulse can be much smaller than the group velocity in vacuum. The group velocity and the group delay are given by:

$$
\begin{gathered}
v_{g}=\frac{c}{n+\omega \frac{d n}{d \omega}} \\
\tau_{g}=L\left(\frac{1}{v_{g}}-\frac{1}{c}\right)
\end{gathered}
$$



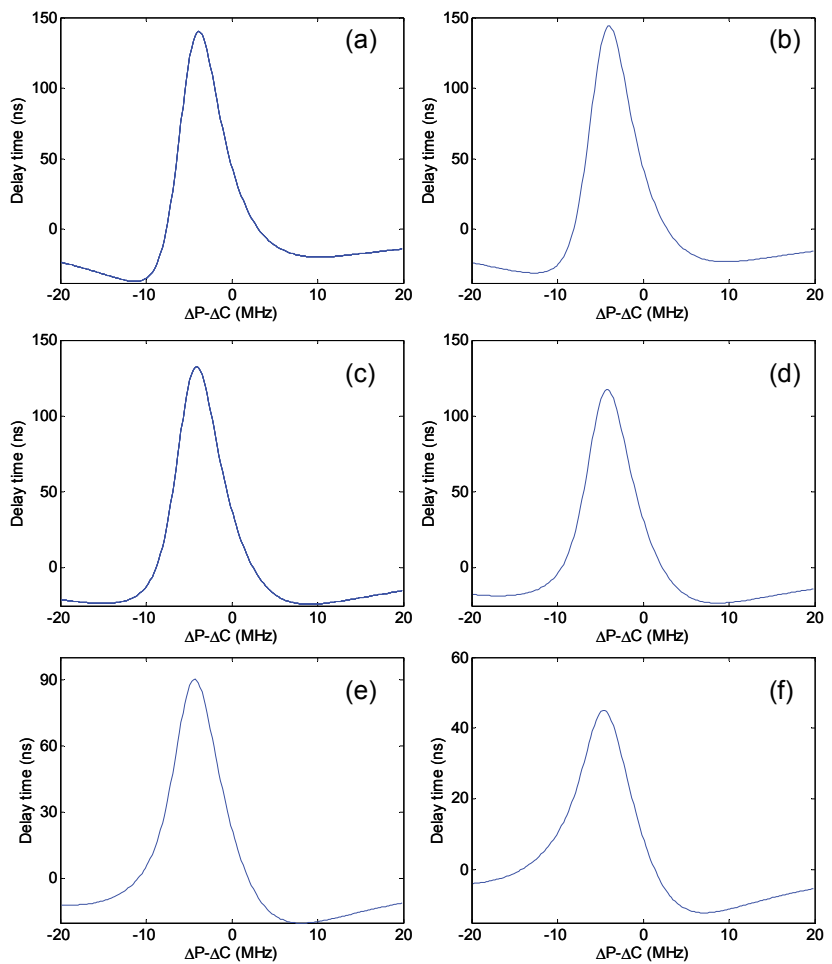

Fig. 7. Group delay time of the probe as a function of the probe detuning for Cases I $\sim$ VI.

where $L$ is the length of the medium, $c$ is the speed of light in vacuum, and $n$ is given by $n=\sqrt{1+\chi^{\prime}}$.

The neighboring excited-state-modified Doppler broadened atoms affect on the EIT line center shifted, resulting in the so-called detuned slow light phenomenon. In Fig. 7 we numerically calculate the group delay of each case mentioned in above, using reasonable parameters according to the actual experimental condition. (Here we choose the same parameters as those in Fig. 6, and let $L=7.5 \mathrm{~cm}$ ).

For all cases, the probe shows a red shift to the slow light. In Fig. 7 (a), for instance, the maximum group delay is red-shifted for the resonant transition by $\sim 4 \mathrm{MHz}$. When the coupling field is tuned to the crossover transitions as shown in Figs. 7(b), 7(d), and 7(e), first, the slow light phenomenon also exists; and second, the maximum group delay position is also detuned from the crossover line center. Even when the coupling field is resonant with the transition $|2>-| 6>$ (Case VI) (transition $|2>-| 6>$ is forbidden to the probe), there also exists slow light and group delay detuning, due to the EIT effects from levels $\mid 4>$ and $|5\rangle$. For more detail information and experimental results see Ref. (Chen et al., 2009).

\section{Slow light in $\mathrm{N}$-type system of ${ }^{87} \mathrm{Rb}$ D2 line}

In this section, we investigate coherent control of the four-level N-type scheme in a Dopplerbroadened six-level atomic system of the ${ }^{87} \mathrm{Rb} \mathrm{D} 2$ line (Chen et al., 2009). With limited spectral distribution of the excited hyperfine states in the ${ }^{87} \mathrm{Rb} \mathrm{D} 2$ line, which is confined 
by the Doppler broadening, each hyperfine state can be used for individual optical channels for optical quantum information processing. For this application we choose nonelectromagnetically induced absorption (EIA) schemes for the investigation of reduced absorption spectra resulting in Mollow sideband-like enhanced transparency windows across the EIT line center. Unlike a double-EIT system satisfied by rigid (uncontrollable) two coupling fields applicable only for a single slow-light channel, the present scheme uses a fixed coupling field with a variable control field, where group velocity control and multiple slow-light channels are applicable.

N-type scheme in a Doppler-broadened six-level atomic system of the ${ }^{87 R b}$ D2 line is shown in Fig. 8. It is similar to EIT situation, but with a third coherent field (the control field) at a frequency $\omega_{S}$ with an amplitude $E_{S}$ couples the transition $|3>-| 1>\left(5 S_{1 / 2}, F=1 \rightarrow 5 \mathrm{P}_{3 / 2}\right.$, $\left.\mathrm{F}^{\prime}=0\right)$ with a detuning of $\Delta_{S}\left(\Delta_{S}=\omega_{31}-\omega_{S}\right)$.

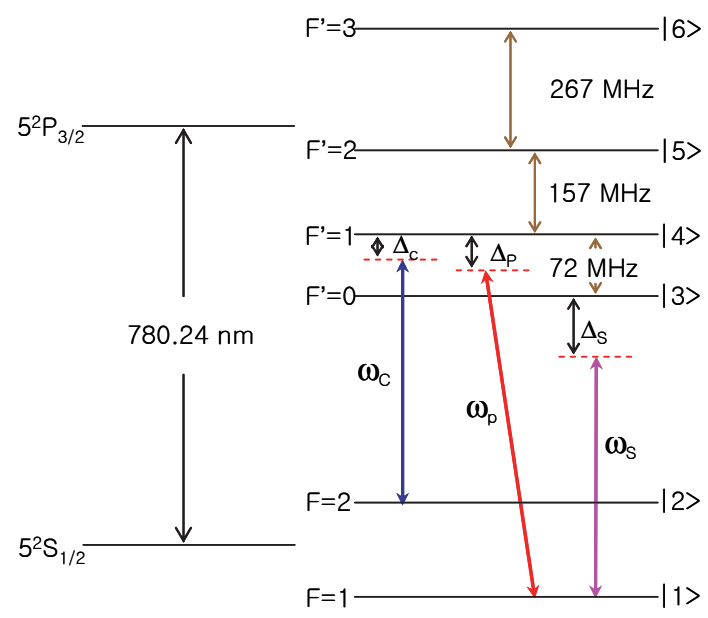

Fig. 8. Schematic of a Doppler-broadened six-level atomic system of the ${ }^{87 \mathrm{Rb}} \mathrm{D} 2$ line interacting with three coherent fields.

In a framework of the semiclassical theory, under the rotating-wave approximation, we obtain the following density matrix equations of motion for the interaction Hamiltonian:

$$
\begin{aligned}
\dot{\rho}_{11}= & \frac{i}{2} \Omega_{S 31}\left(\rho_{31}-\rho_{13}\right)+\frac{i}{2} \Omega_{S 41}\left(\rho_{41}-\rho_{14}\right)+\frac{i}{2} \Omega_{S 51}\left(\rho_{51}-\rho_{15}\right)+\Gamma_{31} \rho_{33} \\
& +\Gamma_{41} \rho_{44}+\Gamma_{51} \rho_{55}+\Gamma_{21} \rho_{22}-\Gamma_{21} \rho_{11}, \\
\dot{\rho}_{12}= & \left(i \Delta_{S}+i \Delta_{34}-i \Delta_{C}-\gamma_{12}\right) \rho_{12}+\frac{i}{2} \Omega_{S 31} \rho_{32}+\frac{i}{2} \Omega_{S 41} \rho_{42}+\frac{i}{2} \Omega_{S 51} \rho_{52} \\
& -\frac{i}{2} \Omega_{C 42} \rho_{14}-\frac{i}{2} \Omega_{C 52} \rho_{15}-\frac{i}{2} \Omega_{C 62} \rho_{16}, \\
\dot{\rho}_{13}= & \left(i \Delta_{S}-\gamma_{13}\right) \rho_{13}+\frac{i}{2} \Omega_{S 31}\left(\rho_{33}-\rho_{11}\right)+\frac{i}{2} \Omega_{S 41} \rho_{43}+\frac{i}{2} \Omega_{S 51} \rho_{53}, \\
\dot{\rho}_{14}= & \left(i \Delta_{S}+i \Delta_{34}-\gamma_{14}\right) \rho_{14}+\frac{i}{2} \Omega_{S 31} \rho_{34}+\frac{i}{2} \Omega_{S 41}\left(\rho_{44}-\rho_{11}\right)+\frac{i}{2} \Omega_{S 51} \rho_{54}-\frac{i}{2} \Omega_{C 42} \rho_{12},
\end{aligned}
$$




$$
\begin{aligned}
& \dot{\rho}_{15}=\left(i \Delta_{S}+i \Delta_{35}-\gamma_{15}\right) \rho_{15}+\frac{i}{2} \Omega_{S 31} \rho_{35}+\frac{i}{2} \Omega_{S 41} \rho_{45}+\frac{i}{2} \Omega_{S 51}\left(\rho_{55}-\rho_{11}\right)-\frac{i}{2} \Omega_{C 52} \rho_{12} ， \\
& \dot{\rho}_{16}=\left(i \Delta_{S}+i \Delta_{36}-\gamma_{16}\right) \rho_{16}+\frac{i}{2} \Omega_{S 31} \rho_{36}+\frac{i}{2} \Omega_{S 41} \rho_{46}+\frac{i}{2} \Omega_{S 51} \rho_{56}-\frac{i}{2} \Omega_{C 62} \rho_{12} \\
& \dot{\rho}_{22}=\frac{i}{2} \Omega_{C 42}\left(\rho_{42}-\rho_{24}\right)+\frac{i}{2} \Omega_{C 52}\left(\rho_{52}-\rho_{25}\right)+\frac{i}{2} \Omega_{C 62}\left(\rho_{62}-\rho_{26}\right)+\Gamma_{42} \rho_{44}+\Gamma_{52} \rho_{55} \\
& +\Gamma_{62} \rho_{66}+\Gamma_{21} \rho_{11}-\Gamma_{21} \rho_{22}, \\
& \dot{\rho}_{23}=\left(i \Delta_{C}-i \Delta_{34}-\gamma_{23}\right) \rho_{23}+\frac{i}{2} \Omega_{C 42} \rho_{43}+\frac{i}{2} \Omega_{C 52} \rho_{53}+\frac{i}{2} \Omega_{C 62} \rho_{63}-\frac{i}{2} \Omega_{S 31} \rho_{21} \text {, } \\
& \dot{\rho}_{24}=\left(i \Delta_{C}-\gamma_{24}\right) \rho_{24}+\frac{i}{2} \Omega_{C 42}\left(\rho_{44}-\rho_{22}\right)+\frac{i}{2} \Omega_{C 52} \rho_{54}+\frac{i}{2} \Omega_{C 62} \rho_{64}-\frac{i}{2} \Omega_{S 41} \rho_{21} \text {, } \\
& \dot{\rho}_{25}=\left(i \Delta_{C}+i \Delta_{45}-\gamma_{25}\right) \rho_{25}+\frac{i}{2} \Omega_{C 52}\left(\rho_{55}-\rho_{22}\right)+\frac{i}{2} \Omega_{C 42} \rho_{45}+\frac{i}{2} \Omega_{C 62} \rho_{65}-\frac{i}{2} \Omega_{S 51} \rho_{21}, \\
& \dot{\rho}_{26}=\left(i \Delta_{C}+i \Delta_{46}-\gamma_{26}\right) \rho_{26}+\frac{i}{2} \Omega_{C 62}\left(\rho_{66}-\rho_{22}\right)+\frac{i}{2} \Omega_{C 42} \rho_{46}+\frac{i}{2} \Omega_{C 52} \rho_{56} \text { ， } \\
& \dot{\rho}_{33}=\frac{i}{2} \Omega_{S 31}\left(\rho_{13}-\rho_{31}\right)-\Gamma_{31} \rho_{33}, \\
& \dot{\rho}_{34}=\left(i \Delta_{34}-\gamma_{34}\right) \rho_{34}+\frac{i}{2} \Omega_{S 31} \rho_{14}-\frac{i}{2} \Omega_{S 41} \rho_{31}-\frac{i}{2} \Omega_{C 42} \rho_{32}, \\
& \dot{\rho}_{35}=\left(i \Delta_{35}-\gamma_{35}\right) \rho_{35}+\frac{i}{2} \Omega_{\mathrm{S} 31} \rho_{15}-\frac{i}{2} \Omega_{\mathrm{S} 51} \rho_{31}-\frac{i}{2} \Omega_{C 52} \rho_{32} \text {, } \\
& \dot{\rho}_{36}=\left(i \Delta_{36}-\gamma_{36}\right) \rho_{36}+\frac{i}{2} \Omega_{S 31} \rho_{16}-\frac{i}{2} \Omega_{C 62} \rho_{32} \text {, } \\
& \dot{\rho}_{44}=\frac{i}{2} \Omega_{S 41}\left(\rho_{14}-\rho_{41}\right)+\frac{i}{2} \Omega_{C 42}\left(\rho_{24}-\rho_{42}\right)-\left(\Gamma_{41}+\Gamma_{42}\right) \rho_{44} ， \\
& \dot{\rho}_{45}=\left(i \Delta_{45}-\gamma_{45}\right) \rho_{45}+\frac{i}{2} \Omega_{S 41} \rho_{15}+\frac{i}{2} \Omega_{C 42} \rho_{25}-\frac{i}{2} \Omega_{S 51} \rho_{41}-\frac{i}{2} \Omega_{C 52} \rho_{42}, \\
& \dot{\rho}_{46}=\left(i \Delta_{46}-\gamma_{46}\right) \rho_{46}+\frac{i}{2} \Omega_{S 41} \rho_{16}+\frac{i}{2} \Omega_{C 42} \rho_{26}-\frac{i}{2} \Omega_{C 62} \rho_{42}, \\
& \dot{\rho}_{55}=\frac{i}{2} \Omega_{S 51}\left(\rho_{15}-\rho_{51}\right)+\frac{i}{2} \Omega_{C 52}\left(\rho_{25}-\rho_{52}\right)-\left(\Gamma_{51}+\Gamma_{52}\right) \rho_{55}, \\
& \dot{\rho}_{56}=\left(i \Delta_{56}-\gamma_{56}\right) \rho_{56}+\frac{i}{2} \Omega_{S 51} \rho_{16}+\frac{i}{2} \Omega_{C 52} \rho_{26}-\frac{i}{2} \Omega_{C 62} \rho_{52} \text {, } \\
& \rho_{i j}=\rho_{j i}, \quad \rho_{11}+\rho_{22}+\rho_{33}+\rho_{44}+\rho_{55}+\rho_{66}=1
\end{aligned}
$$

where $\Omega_{S i 1}=\mu_{i 1} E_{S} / \hbar$ is the Rabi frequency of the control field for the transition $|\mathrm{i}>-| 1>$ $(\mathrm{i}=3,4,5) . \quad \Gamma_{i j}\left(\gamma_{i j}\right)$ stands for the population (phase) decay rate from state $\mid \mathrm{i}>$ to $|\mathrm{j}\rangle$, where $\Gamma_{i 1}$ and $\Gamma_{j 2}$ are the population decay rates from levels $\mid \mathrm{i}>$ to $\mid 1>(\mathrm{i}=3,4,5)$, and levels $\mid j>$ to $\mid 2>(j=4,5,6)$, respectively.

In order to calculate the probe absorption spectrum, the density matrix equations (6) can be rewritten in the following form:

$$
\frac{d \Psi(t)}{d t}=L \Psi(t)+I
$$


where

$$
\begin{aligned}
\Psi= & \left(\rho_{11}, \rho_{12}, \rho_{13}, \rho_{14}, \rho_{15}, \rho_{16}, \rho_{21}, \rho_{22}, \rho_{23}, \rho_{24}, \rho_{25}, \rho_{26}, \rho_{31}, \rho_{32}, \rho_{33}, \rho_{34}, \rho_{35}, \rho_{36},\right. \\
& \left.\rho_{41}, \rho_{42}, \rho_{43}, \rho_{44}, \rho_{45}, \rho_{46}, \rho_{51}, \rho_{52}, \rho_{53}, \rho_{54}, \rho_{55}, \rho_{56}, \rho_{61}, \rho_{62}, \rho_{63}, \rho_{64}, \rho_{65}\right)^{T},
\end{aligned}
$$

and

$$
I=\left(0,0,0,0,0,0,0, \Gamma_{62}, 0,0,0, i \Omega_{\mathrm{C} 62} / 2,0,0,0,0,0,0,0,0,0,0,0,0,0,0,0,0,0,0,0,-i \Omega_{\mathrm{C} 62} / 2,0,0,0\right) .
$$

The solution of Eq. (7) is given by:

$$
\Psi(t)=e^{L\left(t-t_{0}\right)} \Psi\left(t_{0}\right)+\int_{t_{0}}^{t} e^{L\left(t^{\prime}-t 0\right)} I d t^{\prime}
$$

According to the linear response theory, the steady state absorption spectrum of the weak probe laser can be written as:

$$
A\left(\Delta_{P}\right)=\operatorname{Re}\left(\int_{0}^{\infty} \lim _{t \rightarrow \infty}\left\langle\left[P^{-}(t+\tau), P^{+}(t)\right]\right\rangle e^{i \Delta_{P} \tau} d \tau\right)
$$

where $P^{-}=\mu_{31}|1\rangle\left\langle 3\left|+\mu_{41}\right| 1\right\rangle\left\langle 4\left|+\mu_{51}\right| 1\right\rangle\langle 5|$

and $P^{+}=\mu_{31}|3\rangle\left\langle 1\left|+\mu_{41}\right| 4\right\rangle\left\langle 1\left|+\mu_{51}\right| 5\right\rangle\langle 1|$ are the atomic polarization operators, with $\mu_{i 1}$ being the dipole matrix elements of transition $|\mathrm{i}>-| 1>(\mathrm{i}=3,4,5)$. By using the quantum regression theory, we further obtain the absorption and refraction spectra of the probe field:

$$
\begin{aligned}
A\left(\Delta_{P}\right) & =\operatorname{Re}\left[\sum _ { i = 3 } ^ { 5 } \mu _ { i 1 } \left(M_{i, i} \rho_{11}(\infty)+M_{i, 6+i} \rho_{21}(\infty)+M_{i, 12+i} \rho_{31}(\infty)+M_{i, 18+i} \rho_{41}(\infty)\right.\right. \\
& +M_{i, 24+i} \rho_{51}(\infty)+M_{i, 30+i} \rho_{61}(\infty)-\left(M_{i, 1} \rho_{i 1}(\infty)+M_{i, 2} \rho_{i 2}(\infty)\right. \\
& \left.\left.\left.+M_{i, 3} \rho_{i 3}(\infty)+M_{i, 4} \rho_{i 4}(\infty)+M_{i, 5} \rho_{i 5}(\infty)+M_{i, 6} \rho_{i 6}(\infty)\right)\right)\right] \\
B\left(\Delta_{P}\right)= & \operatorname{Im}\left[\sum _ { i = 3 } ^ { 5 } \mu _ { i 1 } ^ { 2 } \left(M_{i, i} \rho_{11}(\infty)+M_{i, 6+i} \rho_{21}(\infty)+M_{i, 12+i} \rho_{31}(\infty)+M_{i, 18+i} \rho_{41}(\infty)\right.\right. \\
& +M_{i, 24+i} \rho_{51}(\infty)+M_{i, 30+i} \rho_{61}(\infty)-\left(M_{i, 1} \rho_{i 1}(\infty)+M_{i, 2} \rho_{i 2}(\infty)+M_{i, 3} \rho_{i 3}(\infty)\right. \\
& \left.\left.+M_{i, 4} \rho_{i 4}(\infty)+M_{i, 5} \rho_{i 5}(\infty)+M_{i, 6} \rho_{i 6}(\infty)\right)\right]
\end{aligned}
$$

where $M=\left(i \Delta_{s}+i \Delta_{34}-i \Delta_{p}-L\right)^{-1}$ and $\left.\rho_{i j}(\infty)\right|_{i, j=1 \rightarrow 35}$ are the steady state solutions of Eq. (6).

The effects of Doppler broadening due to the atom's thermal velocity $v$ can be considered by substituting $\Delta_{P}, \Delta_{S}$ and $\Delta_{C}$ with $\Delta_{P}-\omega_{41} v / c, \Delta_{S}-\omega_{31} v / c$, and $\Delta_{C}-\omega_{42} v / c$, respectively. Then the total absorption and refraction coefficients of the weak probe are:

$$
A\left(\Delta_{P}\right)=\int_{-\infty}^{\infty} A\left(\Delta_{P}, v\right) \frac{N}{v_{p} \sqrt{\pi}} e^{-v^{2} / v_{p}^{2}} d v,
$$




$$
B\left(\Delta_{P}\right)=\int_{-\infty}^{\infty} B\left(\Delta_{P}, v\right) \frac{N}{v_{p} \sqrt{\pi}} e^{-v^{2} / v_{p}^{2}} d v
$$

where $N$ is the total number of atoms, $v_{p}=\sqrt{2 k T / m}=\sqrt{2 R T / M}$ is the most probable atomic velocity, $\mathrm{k}$ is the Boltzmann constant, $\mathrm{R}$ is the gas constant, and $\mathrm{T}$ is the temperature of the atomic system.

Similar as in section 2, we consider the following six types of four-level N-type systems: Type I: The coupling light is resonant with the transition $|2>-| 4>\left(5 \mathrm{~S}_{1 / 2}, \mathrm{~F}=2 \rightarrow 5 \mathrm{P}_{3 / 2}, \mathrm{~F}^{\prime}=1\right)$, while the control light is resonant with the transition $|1>-| 3>\left(5 \mathrm{~S}_{1 / 2}, \mathrm{~F}=1 \rightarrow 5 \mathrm{P}_{3 / 2}, \mathrm{~F}^{\prime}=0\right)$.

Type II: The coupling light is resonant with the transition $|2>-| 4>\left(5 \mathrm{~S}_{1 / 2}, \mathrm{~F}=2 \rightarrow 5 \mathrm{P}_{3 / 2}, \mathrm{~F}^{\prime}=1\right)$, while the control light is resonant with the transition $|1>-| 5>\left(5 \mathrm{~S}_{1 / 2}, \mathrm{~F}=1 \rightarrow 5 \mathrm{P}_{3 / 2}, \mathrm{~F}^{\prime}=2\right)$.

Type III: The coupling light is resonant with the transition $|2>-| 5>\left(5 \mathrm{~S}_{1 / 2}, \mathrm{~F}=2 \rightarrow 5 \mathrm{P}_{3 / 2}, \mathrm{~F}^{\prime}=2\right)$, while the control light is resonant with the transition $|1>-| 4>\left(5 \mathrm{~S}_{1 / 2}, \mathrm{~F}=1 \rightarrow 5 \mathrm{P}_{3 / 2}, \mathrm{~F}^{\prime}=1\right)$.

Type IV: The coupling light is resonant with the transition $|2>-| 5>\left(5 \mathrm{~S}_{1 / 2}, \mathrm{~F}=2 \rightarrow 5 \mathrm{P}_{3 / 2}, \mathrm{~F}^{\prime}=2\right)$, while the control light is resonant with the transition $|1>-| 3>\left(5 S_{1 / 2}, F=1 \rightarrow 5 P_{3 / 2}, F^{\prime}=0\right)$.

Type V: The coupling light is resonant to the center line between states $\mid 4>$ and $|5\rangle$ from state $\mid 2>$, while the control light is resonant with the transition $|1>-| 3>\left(5 \mathrm{~S}_{1 / 2}, \mathrm{~F}=1 \rightarrow 5 \mathrm{P}_{3 / 2}\right.$, $\mathrm{F}^{\prime}=0$ ) with a small detuning $\delta_{1}$.

Type VI: The coupling light is resonant to the center line between states $\mid 5>$ and $\mid 6>$ from state $\mid 2>$, while the control light is resonant with the transition $|1>-| 5>\left(5 \mathrm{~S}_{1 / 2}, \mathrm{~F}=1 \rightarrow 5 \mathrm{P}_{3 / 2}\right.$, $\left.\mathrm{F}^{\prime}=2\right)$ with a small detuning $\delta_{2}$.

Figs. 9 (a), 9 (b), 9 (c), and 9 (d) show the numerical simulation of probe absorption spectra for Type I, Type II, Type III, and Type IV, respectively. Figs. 9(e) $\sim 9$ (h) are energy level diagrams corresponding to Figs. 9(a) $\sim 9$ (d), respectively. The number in parentheses of the coupling $\mathrm{C}$ and control $\mathrm{S}$ stands for relative transition strength of Rabi frequency.

The parameters used in the simulations are $T=25^{\circ} \mathrm{C}, \Gamma_{21}=0.01 \mathrm{MHz}, \Gamma_{31}=\Gamma_{62}=6 \mathrm{MHz}$, $\Gamma_{41}=5 \mathrm{MHz}, \Gamma_{42}=1 \mathrm{MHz}, \Gamma_{51}=\Gamma_{52}=3 \mathrm{MHz}, \triangle_{34}=72 \mathrm{MHz}, \triangle_{45}=157 \mathrm{MHz}, \triangle_{56}=267 \mathrm{MHz}$, $\Omega_{S}=10 \mathrm{MHz}, \Omega_{C 42}=\sqrt{1 / 20} \Omega_{C}, \Omega_{S 41}=\Omega_{S 51}=\sqrt{5 / 12} \Omega_{S}, \Omega_{C}=30 \mathrm{MHz}, \Omega_{S 31}=\sqrt{1 / 6} \Omega_{S}$, $\Omega_{C 52}=\Omega_{C} / 2$ and $\Omega_{C 62}=\sqrt{7 / 10} \Omega_{C}$. The calculations include all level transitions in Fig. 8 . The N-type configuration yields interesting results when two-photon resonance is satisfied between the probe and the coupling for (a) $\triangle_{p}=0 \mathrm{MHz}$, (b) $\triangle_{\mathrm{p}}=0 \mathrm{MHz}$, (c) $\triangle_{\mathrm{p}}=-157 \mathrm{MHz}$, (d) $\triangle p=-157 \mathrm{MHz}$, (e) $\triangle_{\mathrm{p}}=-78.5 \mathrm{MHz}$, and (f) $\triangle_{\mathrm{p}}=-290.5 \mathrm{MHz}$.

In Figs. 9(a) and 9(b), the applied coupling Rabi frequency is much weaker than in Figs. 9(c) and $9(\mathrm{~d})$ by a factor of $\sqrt{5}$. In Figs. 9 (a) and $9(\mathrm{~d})$, the Rabi frequency of the control field is weaker than in Figs. 9(b) and 9(c) by a factor of $\sqrt{5 / 2}$. Thus, Fig. 9(c) is for the strongest pump fields, and a symmetric pair of reduced absorption lines across the EIT line center is obtained (the dotted circle and two arrows indicate the reduced absorption lines): Mollow sideband-like transparency windows. The center transparency is much higher than the satellite transparencies. The symmetric sideband absorption bandwidth is comparable to the EIT linewidth or the spectral hole width. The generation of these absorption-reduced sidebands is due to dynamic energy splitting incurred by the control field acting on the coupling field according to dressed state interactions (Kong et al., 2007):

$$
|D\rangle= \pm \frac{\Omega_{C}^{\prime}}{2} \pm \frac{\Omega_{S}^{\prime}}{2}
$$


where $|\mathrm{D}\rangle$ is the newly developed dressed states by the interaction of the coupling and control fields, and $\Omega_{C}^{\prime}$ and $\Omega_{S}^{\prime}$ are effective Rabi frequencies of the coupling and control fields, respectively, including an atom velocity factor (kv). Fig. 9(d) is similar to Fig. 9(c), also shows double sideband transparency windows. For the rest of the combinations of Figs. 9(a) and 9(b), no distinct change is obtained for the Mollow sideband-like transparency windows because of a weak field limit.

In comparison with Fig. 3(c) of Ref. (Kong et al., 2007), where the probe gain results in, rather than the Mollow sideband-like transparency, Fig. 9(c) here needs to be analyzed in more detail (see Fig. 11). Moreover the origin of the Mollow sideband-like effects which appeared in Fig. 4(a) of Ref. (Kong et al., 2007) for the case of $F_{e}=F_{g}+1$ by using D2 transition for the coupling but using D1 transition for the control, is the same as in Fig. 9(c) of the present chapter for the case of $\mathrm{F}_{\mathrm{e}} \leq \mathrm{F}_{\mathrm{g}}$ by using only $\mathrm{D} 2$ transition for both fields under the EIT condition. This condition will be discussed in Fig. 11 below.

According to Eq. (12), EIA-like enhanced absorption should be possible if $\Omega_{C}=\Omega_{S}$ (see Fig. 11(c)), owing to degenerate dressed states at the EIT line center. The sub-Doppler ultranarrow double transparency windows obtained in Fig. 9(c) have the potential of using double ultraslow light pulses for optical and quantum information processing such as Schrödinger's cat generation or quantum gate operation. For enhanced cross-phase modulation, double EIT-based ultraslow light is required. Multichannel all-optical buffer memory is another potential application.

Fig. 10 shows numerical simulation results of an absorption spectrum when the coupling laser $\Omega_{C}$ is tuned to crossover lines, which is a line center between levels $|4\rangle$ and $|5\rangle$ for Fig. 10(a) and |5> and |6> for Fig. 10(b): Types V and VI, respectively. In each case the Mollow sideband-like transparency windows appear. The control is purposely detuned by 6 $\mathrm{MHz}$ for Fig. 10 (a) for the transition $|1>\leftrightarrow| 3>\left(5 \mathrm{~S}_{1 / 2}, \mathrm{~F}=1 \rightarrow 5 \mathrm{P}_{3 / 2}, \mathrm{~F}^{\prime}=0\right)$, and $30 \mathrm{MHz}$ for Fig. 10 (b) for $|1>\leftrightarrow| 5>\left(5 S_{1 / 2}, F=1 \rightarrow 5 P_{3 / 2}, F^{\prime}=2\right)$. As shown in Fig. 10, the results are very similar to Fig. 9(c). The Mollow sideband-like reduced absorption lines and the hole-burning peak also appears on the right.

We now analyze Fig. 10 as follows, using the velocity selective atoms phenomenon. The original model of Fig. 10(a) can be divided into two models, as shown in the energy level diagram just below Fig. 10. The first row is for Fig. 10(a), and the second row is for Fig. 10(b). The left column is for the original level transition, and the right two columns are decomposed for purposes of analysis. For these two columns of energy-level diagrams, blue-Doppler-shifted atoms (middle column) and red-Doppler-shifted atoms (right column) by $\Delta_{1}=78.5 \mathrm{MHz}$ or $\Delta_{2}=133.5 \mathrm{MHz}$ are considered.

In the first row (for Fig. 10(a)) for blue-Doppler-shifted atoms (middle column), the blue shift $\triangle(\triangle=157 / 2=78.5 \mathrm{MHz})$ makes both the coupling field (C) and the control field (S) (see the middle column) resonant. This result occurs because initially the control field is red detuned by $\delta_{1}(6 \mathrm{MHz})$; thus the total shift is $72.5 \mathrm{MHz}(78.5-6)$, which is nearly resonant to the transition of $|1>\leftrightarrow| 4>$. This outcome is the same as in Fig. 9(c). The right column, however, does not form an N-type model because of a big detuning of $\Delta_{1}+\delta_{1}$. The EIT window cannot be affected by the detuning $\Delta_{1}+\delta_{1}$ if two-photon resonance is satisfied. Actually, signal reduction and line narrowing result, but do not affect the line shape of Fig. 10(a). Therefore, the result of Fig. 10(a) must be the same as for Fig. 9(c). 

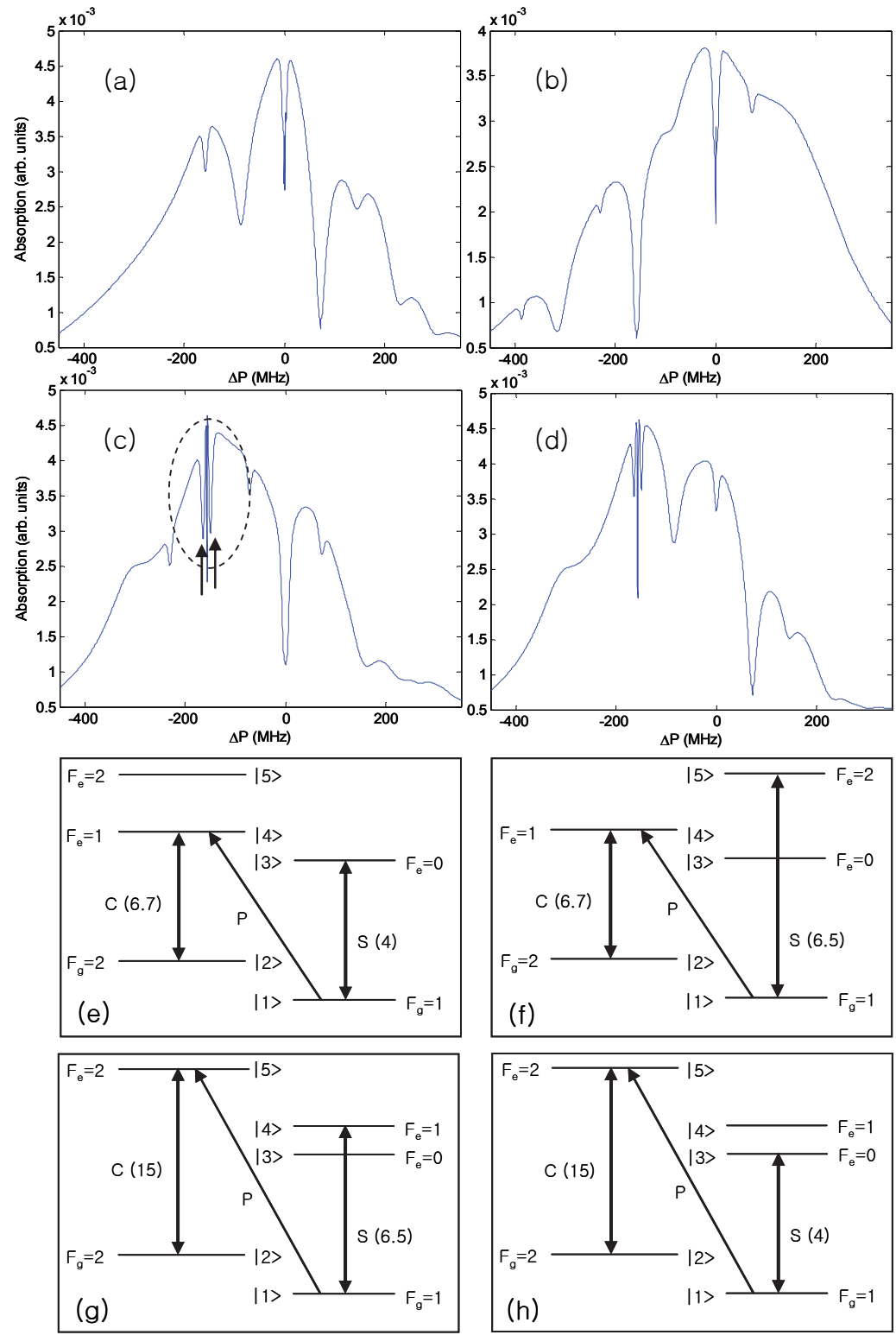

Fig. 9. Numerical calculations for the probe absorption for Type I, Type II, Type III, and Type IV. 

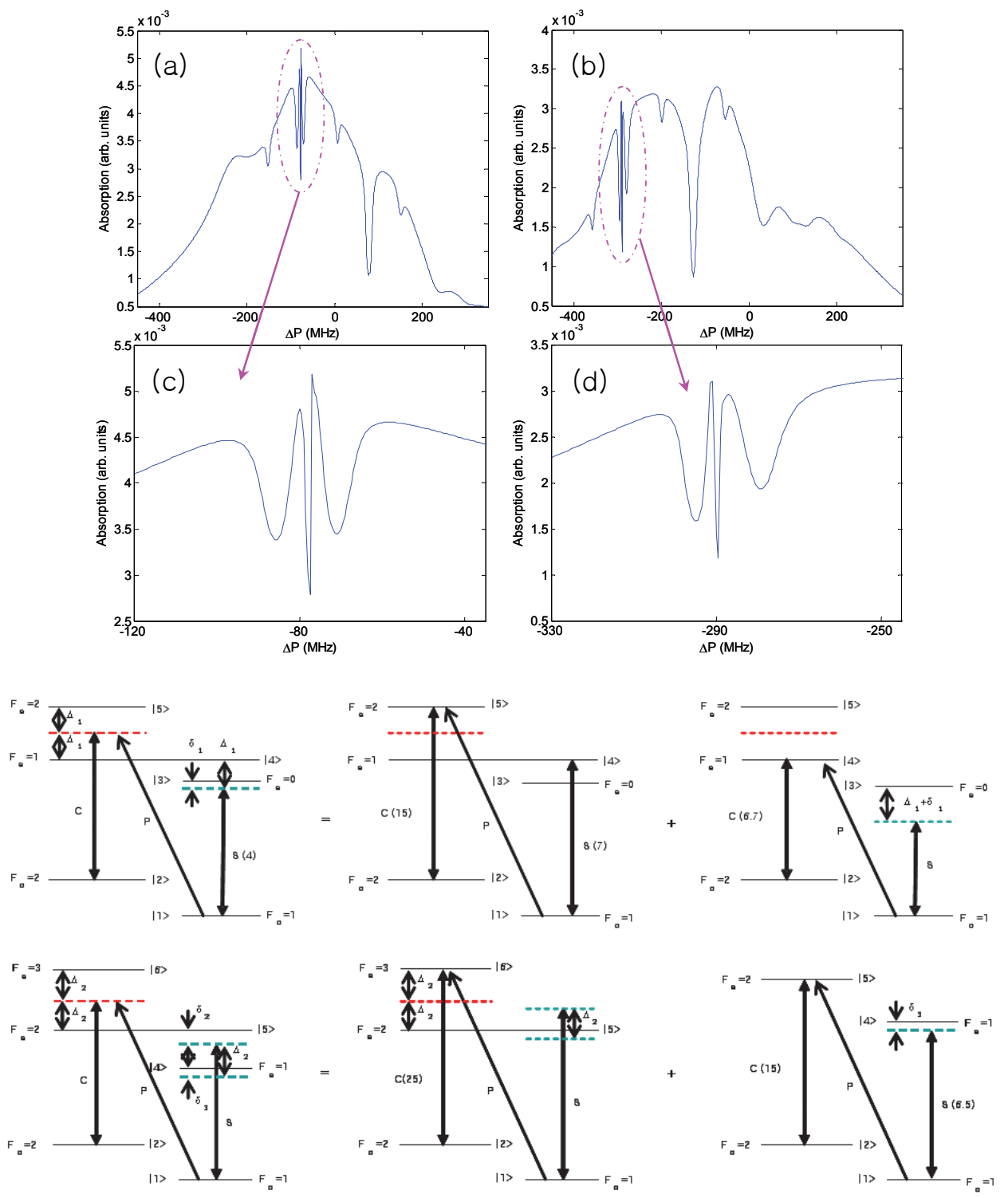

Fig. 10. Numerical calculations for the probe absorption for (a) Type V and (b) Type VI. (c) and (d) are for the extended feature of (a) and (b) , respectively.

In the second row (for Fig. 10(b)), for red-Doppler-shifted atoms (right column), the red shift $\triangle(\triangle=-267 / 2=-133.5 \mathrm{MHz})$ makes the coupling field (C) resonant, but blue detuned to the control field (S) by $23.5 \mathrm{MHz}$. However, the control field is set to be red detuned by $\delta_{2}$ (30 $\mathrm{MHz})$ initially; the net detuning is $\delta_{3}(6.5 \mathrm{MHz})$ to the control, which is red detuned from 
the transition of $|1>\leftrightarrow| 4>$. The two sidebands across the EIT line center are asymmetric. The blue-Doppler-shifted atoms (middle column) do not contribute anything on the sideband transparency windows as discussed for the first row because of too much detuning of the control field to form an N-type model.

Figs. 10(c) and 10(d) represent expanded absorption spectra of Figs. 10(a) and 10(b), respectively. As discussed in above, Fig. 10(c) is for resonant transition, and Fig. 10(d) is for off-resonant transition to the control field. As shown, the off-resonant case generates asymmetric Mollow sideband-like transparency windows with unequal window linewidth. Hence each probe light group velocity at each sideband can be controlled effectively with on-demand detuning of the control field. For the cross-phase modulation, this controllability is important to induce on-demand $\pi$ phase shift (Petrosyan \& Kurizki, 2002; Paternostro et al., 2003).

Fig. 11 represents the probe absorption spectrum versus the control (S) Rabi frequency for a fixed coupling $(\mathrm{C})$ Rabi frequency and population decay rate $\Gamma_{42}$ (from the excited state |4> to the ground state $\mid 2>$ ) in a closed N-type model of Fig. 9(g). In the closed N-type model of Fig. $9(\mathrm{~g})$, the atom flow rate of circulation at the probe line center should depend on both $\Gamma_{42}$ and the control field strength. For a fast (slow) $\Gamma_{42}$, the probe experiences fast circulation and has more change on the probe spectrum. On the other hand, as seen in Fig. 11(c), the dressed state interactions at a low decay rate of $\Gamma_{42}=1 \mathrm{MHz}$ results in enhanced absorption at the probe line center when the coupling and the control Rabi frequencies are equal (Kong et al., 2007). As shown in Figs. 11(a) 11(c) for a weak decay rate, the probe gain may not be possible regardless of the control field strength because no population inversion between states $|5\rangle$ and $|1\rangle$ can be obtained. Applying moderate control strength (see the center column), however, one can obtain the probe gain once the system is ready for a fast atom flow rate, for example, with a high decay rate of $\Gamma_{42}=10 \mathrm{MHz}$ (see Fig. 11(h)). Thus, the probe gain or EIT-like absorption must be understood in terms of system parameters of both control field strength and the medium's decay rate.

For balanced Rabi frequency between the coupling and the control, the EIA-like enhanced absorption can be obtained in Fig. 11(c). However, this enhanced absorption feature, which resulted from degeneracy of the dressed states (see Eq. (12)), changes into a probe gain if the atom flow rate increases as shown in Fig. 11(f) (see also Fig. 3(c) of Ref. (Kong et al., 2007)). The observation in Fig. $9(\mathrm{~b})$ is for the intermediate case: $\Omega_{C} \sim \Omega_{S}$ and $1 \mathrm{MHz}<\Gamma_{52}=3 \mathrm{MHz}$ $<5 \mathrm{MHz}$ (see Fig. 9(f)). The decay rate falling between Figs. 11(c) and 11(f) explains a transient feature from the EIA-like absorption to the probe gain as seen in Fig. 9(b). We think that the broadened linewidth of the red line at $\triangle_{p}=0$ in Fig. 9(b), may be caused by this intermediate feature with laser jitter as well as a weak control field. As numerically demonstrated in Fig. 11, the probe gain may not be possible in any types of the ${ }^{87 \mathrm{Rb} D}$ 2 line in Fig. 9 because the atom flow rate is not fast enough (see $\Gamma_{42}=1 \mathrm{MHz}$ in Fig. 9(c)) unless a very strong control field is applied. The formation of Mollow sideband-like transparency windows in Fig. 4 of Ref. (Kong et al., 2007) and Fig. 9(c) of this chapter shows a very similar feature based on the dressed state interactions. However, Ref. (Kong et al., 2007) is not for EIT, while the present scheme is.

By using the Eq. (11-2), we can get the refraction coefficient of the probe. Because of the steep dispersion spectrum resulting from EIT window and sideband-like 
transparency windows, we can get slow light in three channels. The group velocity is given by Eq. (4).
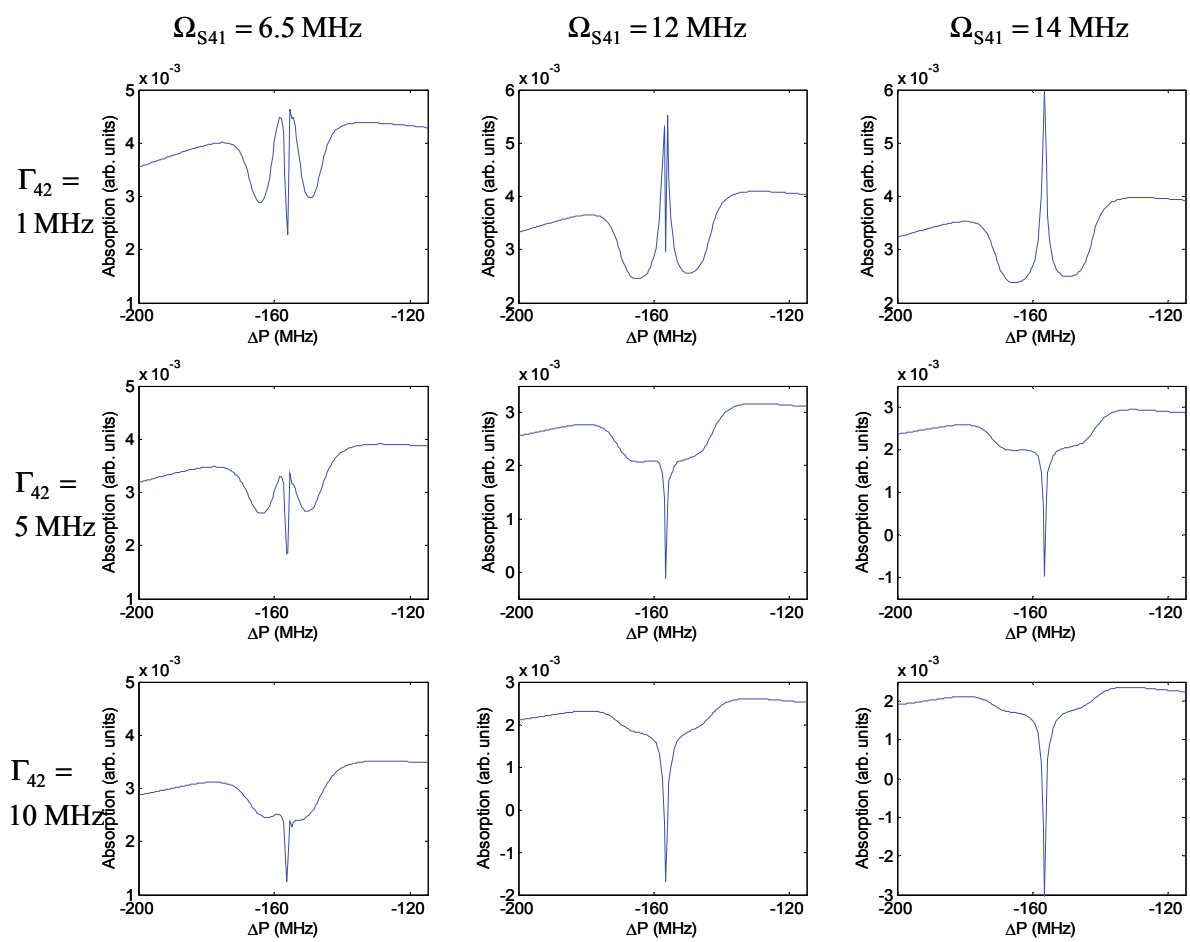

Fig. 11. Probe absorption spectra versus $\Gamma_{42}$ and $\Omega_{S}$ for the case of Fig. $9(\mathrm{~g})$. (a) The expanded feature of Fig. 9(c). (c) EIA-like enhanced absorption. (f), (h), (i): probe gain.

For the fixed atomic density, the width and depth of EIT widow are mainly depending on the intensity (Rabi frequency) of the coupling laser. The dipole moment of transition $|2\rangle \leftrightarrow|5\rangle$ is larger than the dipole moment of transition $|2\rangle \leftrightarrow \mid 4>$ by a factor $\sqrt{5}$, this means we have large slow light when the coupling laser resonant with transition $|2>\leftrightarrow| 4>$. However, as shown in previous part, we can get more obviously multichannel slow light phenomena when coupling light resonant with the transition $|2>\leftrightarrow| 5>$ due to the dipole moment and decay rate relationship in $87 \mathrm{Rb}$ D2 line. Fig. 12 shows the refractive index and group index as a function of the detuning of the probe for different Rabi frequency of the control field. As seen in Fig. 11 and Fig. 12, the separation between two peaks of the Mollow sideband-like transparency windows is invariant for the control field intensity, which means the coupling Rabi frequency $\mathrm{C} \square$ determines the splitting. The linewidth of the transparency windows, however, is controllable by adjusting the control field intensity or its detuning (see Figs 10(c) and 10(d)). Thus, the group velocity of the probe light at the sidebands is also controllable. That means double 
slow light-based enhanced cross-phase modulation is applicable in a much simpler scheme than the scheme suggested in Ref. (Kong et al., 2007). By the way, applications of the enhanced cross-phase modulation are also applicable to the EIT center line and the spectral hole-burning line.
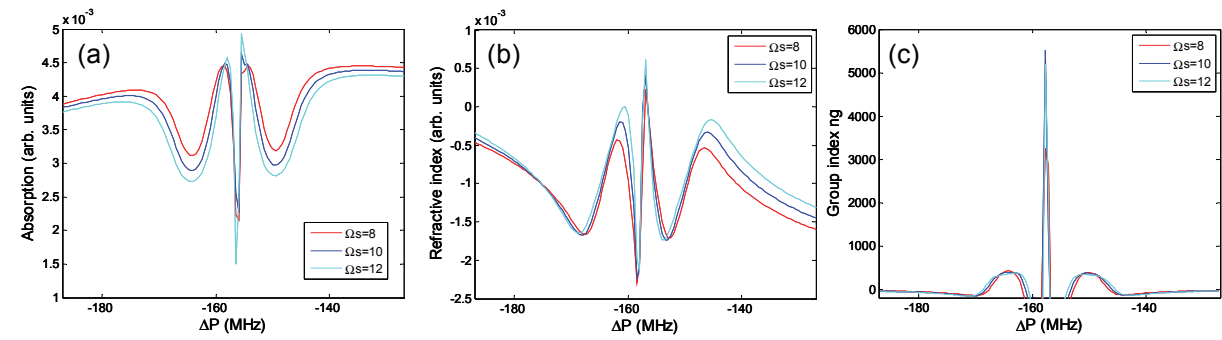

Fig. 12. (a) Absorption, (b) dispersion and (c) group index of the probe as a function of the detuning of the probe for Type III for different Rabi frequency of control light. $\mathrm{N}=10^{10} \mathrm{~cm}^{-3}$.

\section{Conclusions}

We have investigated EIT and EIT-based slow light in a Doppler-broadened six-level atomic system of ${ }^{87} \mathrm{Rb}$ D2 line. The EIT dip shift due to the existence of the neighbouring levels has been numerically analyzed. When the coupling field is tuned to the different transition, we have shown the dependence of group delay of the one-photon detuning of the probe. Based on the EIT study, we also have discussed several N-type schemes in such system. The obtained Mollow sideband-like transparency windows across the EIT line centre are subDoppler broadened and controllable by adjusting the control field intensity or detuning. The work in this chapter may deepen the understanding of EIT and the slow light phenomenon in multilevel system and lead to potential applications in the use of ultraslow light for optical information processing such as all-optical multichannel buffer memory and quantum gate based on enhanced cross-phase modulation owing to increased interaction time between two slow-light pulses.

\section{Acknowledgements}

This work was supported by the Fund of Jilin University and also supported by the Creative Research Initiative program (Center for Photon Information Processing) of MEST via KOSEF.

\section{References}

Alzetta, G.; Gozzini, A.; Moi, L. \& Oriols, G. (1976). An experimental method for the observation of r.f. transitions and laser beat resonances in oriented sodium vapor. Nuovo Cimento B, Vol. 36, 5-20. 
Arimondo, E. \& Orriols, G. (1976). Nonabsorbing atomic coherences by coherent two-photon transitions in a three-level optical pumping. Nuovo Cimento Letters, Vol. 17, No. 6, 333-338.

Boller, K. -J; Imamolu, A. \& Harris, S. E. (1991). Observation of electromagnetically induced transparency. Phys. Rev. Lett., Vol. 66, No. 20, 2593-2596, ISSN 00319007.

Boyd, R. W. \& Gauthier, D. J. (2002). 'Slow' and 'fast' light. Progress in Optics 43, edited by E. Wolf, Chap. 6, 497 (Elsevier, Amsterdam).

Chen, Y.; Wei, X. G. \& Ham, B. S. (2009). Detuned slow light in the Doppler broadened multi-level D2 line of Rubidium. Optics Express, Vol. 17, No. 3, 1781-1788.

Chen, Y.; Wei, X. G. \& Ham, B. S. (2009). Optical properties of an N-type system in Dopplerbroadened multilevel atomic media of the rubidium D2 line. J. Phys. B: At. Mol. Opt. Phys., Vol. 42, 065506.

Fleischhauer, M.; Imamoglu, A. \& Marangos, J. P. (2005). Electromagnetically induced transparency: optics in coherent media. Rev. Mod. Phys., Vol. 77, No. 2, 633-673, ISSN 0034-6861.

Gray, H. R.; Whitley, R. M. \& Stroud, Carlos R., Jr. (1978). Coherent trapping of atomic populations. Opt. Lett., Vol. 3, 218-220, ISSN 0146-9592.

Ham, B. S. (2008). Observation of delayed all-optical routing in a slow-light regime. Phys. Rev. A, Vol. 78, 011808 (R).

Ham, B. S.; Hemmer, P. R. \& Shahriar, M. S. (1997). Efficient electromagnetically induced transparency in a rare-earth doped crystal. Opt. Commun., Vol. 144, 227-230, ISSN 0030-4018.

Harris, S. E. (1989). Lasers without inversion: Interference of lifetime-broadened resonances. Phys. Rev. Lett., Vol. 62, No. 9, (1989) 1033-1036, ISSN 0031-9007

Harris, S. E. (1997). Electromagnetically induced transparency. Phys. Today, Vol. 50, No. 7, 36-42; ISSN 0031-9228.

Harris, S. E.; Fieldm, J. E. \& Imamoglu, A. (1990). Nonlinear optical processes using electromagnetically induced transparency. Phys. Rev. Lett., Vol. 64, No. 10, 11071110, ISSN 0031-9007.

Harris, S. E.; Fieldm, J. E. \& Kasapi, A. (1992). Dispersive properties of electromagnetically induced transparency. Phys. Rev. A, Vol. 46, R29-32.

Hau, L. V.; Harris, S. E; Dutton, Z. \& Behroozi. (1999). Light speed reduction to 17 metres per second in an ultracold atomic gas. Nature, Vol. 397, 594-598, ISSN 00280836.

Julsgaard, B.; Sherson, J.; Cirac, J. I.; Fiurasek, J. \& Polzik, E. S. (2004). Experimental demonstration of quantum memory for light. Nature. Vol. 42, 482.

Kasapi, A.; Jain, M.; Yin, G. Y. \& Harris, S. E. (1995). Electromagnetically induced transparency: propagation dynamics. Phys. Rev. Lett., Vol. 74, No. 13, 2447-2450, ISSN 0031-9007.

Kash, M. M.; Sautenkov, V. A.; Zibrov, A. S.; Hollberg, L.; Welch, G. R.; Lukin, M. D.; Rostovtsev, Y.; Fry, E. S. \& Scully, M. O. (1999). Ultraslow group velocity and enhanced nonlinear optical effects in a coherently driven hot atomic gas. Phys. Rev. Lett., Vol. 82, 5229, ISSN 0031-9007. 
Kong, L. B.; Tu, X. H.; Wang, J. ; Zhu, Y. F.\& Zhan, M. S. (2007). Sub-Doppler spectral resolution in a resonantly driven four-level coherent medium. Opt. Commun., Vol. $269,362$.

Liu, C.; Dutton, Z.; Behroozi, C. H.; Hau, L. V. \& Harris, S. E. (2001). Observation of coherent optical information storage in an atomic medium using halted light pulses. Nature, Vol. 409, 490-493, ISSN 0028-0836.

Lukin, M. D. \& Hemmer, P. R. (2000). Quantum entanglement via optical control of atomatom interactions. Phys. Rev. Lett., Vol. 84, 2818.

Marangos, J. P. (1998). Topical review electromagnetically induced transparency. J. Modern Opt., Vol. 45, No. 3, 471-503, ISSN 0950-0340.

Nielsen, M. A. \& Chuang, I. L. (2000). Quantum Computation and Quantum Information. Cambridge University Press, Cambridge, ISBN 0521635039.

Padmabandu, G. G.; Welch, G. R.; Shubin, I. N.; Fry, E. S.; Nikonov, D. E.; Lukin, M. D. \& Scully, M. O. (1996). Laser oscillation without population inversion in a sodium atomic beam. Phys. Rev. Lett., Vol. 76, No. 12, 2053-2056, ISSN 00319007.

Paternostro, M.; Kim, M. S. \& Ham, B. S. (2003). Generation of entangled coherent states via cross-phase-modulation in a double electromagnetically induced transparency regime. Phys. Rev. A, Vol. 67, 023811.

Petrosyan, D. \& Kurizki, G. (2002). Symmetric photon-photon coupling by atoms with Zeeman-split sublevels. Phys. Rev. A, Vol. 65, 033833.

Phillips, D. F.; Fleischhaueer, A.; Mair, A. \& Walsworth, R. L. (2001). Storage of light in atomic vapor. Phys. Rev. Lett., Vol. 86, 783, ISSN 0031-9007.

Phillips, M. C.; Wang, H. L.; Rumyantsev, I.; Kwong, N. H.; Takayama, R. \& Binder, R. (2003). Electromagnetically induced transparency in semiconductors via biexcition coherence. Phys. Rev. Lett., Vol. 91, No. 18, 183602, ISSN 00319007.

Serapiglia, G. B.; Paspalakis, E.; Sirtori, C.; Vdopyanov, K. L. \& Phillips, C. C. (2000). Laserinduced quantum coherence in a semiconductor quantum well. Phys. Rev. Lett., Vol. 84, 1019, ISSN 0031-9007.

Schmidt, H. \& Imamoglu, A. (1996). Giant Kerr nonlinearities obtained by electromagnetically induced transparency. Opt. Lett., Vol. 21, 1936-1938, ISSN 01469592.

Scully, M. O.; Zhu, S. Y. \& Gravrielides, A. (1989). Degenerate quantum-beat laser: Lasing without inversion and inversion without lasing. Phys. Rev. Lett., Vol. 62, No.24, 2813-2816, ISSN 0031-9007.

Scully, M. O. (1991). Enhancement of the index of refraction via quantum coherence. Phys. Rev. Lett., Vol. 67, No. 14, 1855-1858, ISSN 0031-9007.

Scully, M. O. \& Zhu, S. Y. (1992). Ultra-large index of refraction via quantum interference. Opt. Commun., Vol. 87, 134-138, ISSN 0030-4018.

Scully, M. O. \& Zubairy, M. S. (1997). Quantum Optics. Cambridge University Press, Cambridge, England, ISBN 0521435951.

Turukhin, A. V.; Sudarshanam, V. S; Shahriar, M. S.; Musser, J. A.; Ham, B. S. \& Hemmer, P. R. (2002). Observation of ultraslow and stored light pulses in a solid. Phys. Rev. Lett., Vol. 88, No. 2, 023602, ISSN 0031-9007. 
Xiao, M. \& Li, Y. Q. (1995). Electromagnetically induced transparency in a threelevel lambda type system in rubidium atoms. Phys. Rev. A, Vol. 51, R27032706.

Xiao, M.; Li, Y. Q.; Jin, S. Z. \& Gea-Banacloche, J. (1995). Measurement of dispersive properties of electromagnetically induced transparency in rubidium atom. Phys. Rev. Lett., Vol. 74, 666-669, ISSN 0031-9007. 


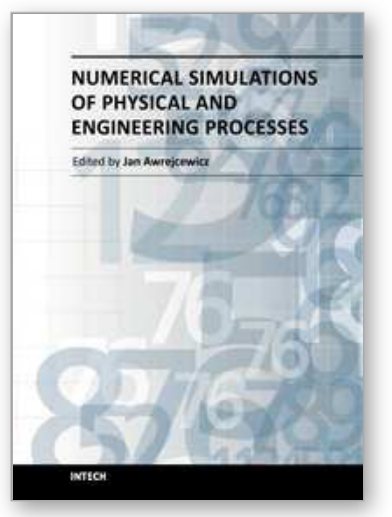

\author{
Numerical Simulations of Physical and Engineering Processes \\ Edited by Prof. Jan Awrejcewicz
}

ISBN 978-953-307-620-1

Hard cover, 594 pages

Publisher InTech

Published online 26, September, 2011

Published in print edition September, 2011

Numerical Simulations of Physical and Engineering Process is an edited book divided into two parts. Part I devoted to Physical Processes contains 14 chapters, whereas Part II titled Engineering Processes has 13 contributions. The book handles the recent research devoted to numerical simulations of physical and engineering systems. It can be treated as a bridge linking various numerical approaches of two closely interrelated branches of science, i.e. physics and engineering. Since the numerical simulations play a key role in both theoretical and application oriented research, professional reference books are highly needed by pure research scientists, applied mathematicians, engineers as well post-graduate students. In other words, it is expected that the book will serve as an effective tool in training the mentioned groups of researchers and beyond.

\title{
How to reference
}

In order to correctly reference this scholarly work, feel free to copy and paste the following:

Yi Chen, Xiao Gang Wei and Byoung Seung Ham (2011). Numerical Simulation of ElT-Based Slow Light in the Doppler-Broadened Atomic Media of the Rubidium D2 Line, Numerical Simulations of Physical and Engineering Processes, Prof. Jan Awrejcewicz (Ed.), ISBN: 978-953-307-620-1, InTech, Available from: http://www.intechopen.com/books/numerical-simulations-of-physical-and-engineering-processes/numericalsimulation-of-eit-based-slow-light-in-the-doppler-broadened-atomic-media-of-the-rubidium-d

\section{INTECH}

open science | open minds

\author{
InTech Europe \\ University Campus STeP Ri \\ Slavka Krautzeka 83/A \\ 51000 Rijeka, Croatia \\ Phone: +385 (51) 770447 \\ Fax: +385 (51) 686166 \\ www.intechopen.com
}

\author{
InTech China \\ Unit 405, Office Block, Hotel Equatorial Shanghai \\ No.65, Yan An Road (West), Shanghai, 200040, China \\ 中国上海市延安西路65号上海国际贵都大饭店办公楼 405 单元 \\ Phone: +86-21-62489820 \\ Fax: +86-21-62489821
}


(C) 2011 The Author(s). Licensee IntechOpen. This chapter is distributed under the terms of the Creative Commons Attribution-NonCommercialShareAlike-3.0 License, which permits use, distribution and reproduction for non-commercial purposes, provided the original is properly cited and derivative works building on this content are distributed under the same license. 